\title{
High throughput clinical CMR service: role of technologists in LV volume analysis
}

\author{
Elisa McAlindon*, Chris Lawton, Cornelius Imobeke, Jessica Harris, Barney Reeves, Chiara Bucciarelli-Ducci \\ From 15th Annual SCMR Scientific Sessions \\ Orlando, FL, USA. 2-5 February 2012
}

\section{Summary}

This study investigates the role of CMR technologists in performing LV volume analysis in a busy clinical CMR service.

\section{Background}

Cardiac magnetic resonance (CMR) imaging studies are increasingly being used in clinical practice for assessment of viability following an acute coronary syndrome. There have been no previous studies investigating the role of CMR technologists in contributing to the analysis of these scans using semi-automated software.

\section{Methods}

20 CMR studies of acute coronary syndromes were included in this study. Analysis was conducted using Argus, Siemens semi-automated volume analysis software. 3 observers conducted the image analysis with two levels of training: Observer 1) "Reference" CMR level 3 physician; Observer 2) and 3) CMR technologists with no previous experience on CMR analysis. Both technologists underwent a $2 \mathrm{~h}$ tutorial on how to use the software. All 3 observers analysed 20 studies, and reanalysed 10 studies 24 hours after.

\section{Results}

Intra-observer variability was assessed using intraclass correlation coefficient (ICC); inter-observer variability was assessed using Bland Altman plots for agreement.

We observed a good inter-observer variability between Observer 1 and 2, and between Observer 1 and 3 in assessing EDV, ESV, and LV mass (Table 1). Among these parameters, the lowest inter-observer variability was observed for ESV. Bland Altman plots suggested acceptable agreement.
Technologist intra-observer variability was comparable to that of the reference observer (ICC technologist volumes and mass 0.94-0.99, ICC reference 0.97-0.99).

\section{Conclusions}

Following a short training, CMR technologists can perform an accurate analysis of LV volume using semiautomated software, compared to an expert CMR physician. These results suggest that technologists can play a role in assisting clinical reporting in a busy clinical CMR service.

\section{Funding}

NIHR Cardiovascular BRU, Bristol Heart Institute.

Table 1 Inter-observer variability

\begin{tabular}{lcccc}
\hline & Observer & EDV & ESV* & Mass \\
\hline $\mathbf{1}$ vs $\mathbf{2}$ & Mean (SD) difference & $15.1(10.5)$ & $0.1(0.2)$ & $20.6(14.2)$ \\
& Mean (SD) & $154.7(31.4)$ & $4.3(0.4)$ & $140.6(29.2)$ \\
& Coefficient of variability (\%) & 6.8 & 4.1 & 10.1 \\
\hline $\mathbf{1}$ vs 3 & Mean (SD) difference & $17.4(10.9)$ & $0.4(0.2)$ & $25.6(14.1)$ \\
& Mean (SD) & $155.9(31.3)$ & $4.4(0.4)$ & $144.5(30.0)$ \\
& Coefficient of variability (\%) & 7.0 & 4.7 & 9.8 \\
\hline
\end{tabular}

Summary statistics calculated are mean and SD of differences, mean and SD of values. Differences between observers are assessed using Bland-Altman plots. *log-transformed for skewed distribution.

Published: 1 February 2012

\section{doi:10.1186/1532-429X-14-S1-P44}

Cite this article as: McAlindon et al:: High throughput clinical CMR service: role of technologists in LV volume analysis. Journal of Cardiovascular Magnetic Resonance 2012 14(Suppl 1):P44.

Bristol Heart Institute, NIHR Cardiovascular BRU, Bristol, UK 